\title{
Malignant transformation of pleomorphic xanthoastrocytoma and differential diagnosis: case report
}

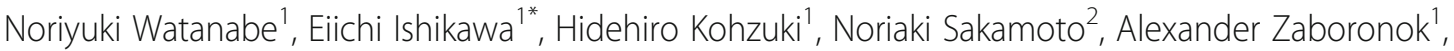 \\ Masahide Matsuda', Makoto Shibuya ${ }^{3}$ and Akira Matsumura ${ }^{1}$
}

\begin{abstract}
Background: Pleomorphic xanthoastrocytoma (PXA) is a rare astrocytic glioma, characterized by large pleomorphic and frequently multinucleated cells, spindle and lipidized cells, a dense pericellular reticulin network, and numerous eosinophilic granular bodies according to the grade II glial tumor standards of the World Health Organization's (WHO) 2016 guidelines. PXA rarely transforms into anaplastic PXA or glioblastoma (GBM) and anaplastic PXA, classified as WHO grade III, has a more aggressive clinical behavior with poorer prognosis than PXA.

Case presentation: Here we describe an unusual case of PXA in a 19-year-old woman, first admitted with headache and a mass in the left temporal lobe in 2005 that was removed. Twelve years later, she returned with left temporal headache, diplopia and tinnitus. A local tumor recurrence was found, and a second resection was performed. The specimen showed highly malignant findings, such as necrosis, microvascular proliferation, and multiple mitoses. The integrated diagnosis was made as high grade glioma, probably derived from PXA. Immunohistochemical (IHC) stains were positive for oligo2, and approximately 21\% positive for Ki-67, while negative for CD34, IDH1 R132H. INI1 and ATRX were retained. As the histological classification was glioblastoma, the patient received GBM-appropriate chemotherapy and radiation therapy and outpatient follow-ups have demonstrated no obvious symptoms for 1 year after surgery. Additional molecular analyses found BRAF V600E mutations in both resections, supporting the idea that the recurrent tumor had derived from PXA.

Conclusions: This case highlights the complexities of differential diagnosis based on the World Health Organization's 2016 guidelines. More integrated criteria to differentiate anaplastic PXA from GBM and epithelioid GBM, combined with genetic screening results, might be needed.
\end{abstract}

Keywords: Pleomorphic xanthoastrocytoma, Malignant transformation, Glioblastoma, Tumor recurrence, Differential diagnosis

\section{Background}

Pleomorphic xanthoastrocytoma (PXA) is a rare astrocytic glioma, characterized by large pleomorphic and frequently multinucleated cells, spindle and lipidized cells, a dense pericellular reticulin network, and numerous eosinophilic granular bodies (EGBs) according to the grade II glial tumor standards of the World Health Organization's (WHO) 2016 guidelines [1]. PXA rarely transforms into anaplastic pleomorphic xanthoastrocytoma (APXA)

\footnotetext{
*Correspondence: e-ishikawa@md.tsukuba.ac.jp

'Departments of Neurosurgery, Faculty of Medicine, University of Tsukuba,

1-1-1 Tennodai, Tsukuba, Ibaraki, Japan

Full list of author information is available at the end of the article
}

or glioblastoma (GBM). APXA, classified as WHO grade III, has a more aggressive clinical behavior with poorer prognosis than PXA [2, 3]. Histologically, APXA is defined when, in addition to PXA, mitoses number 5 or more per high-power field while GBM displays nuclear atypia, cellular pleomorphism, mitotic activity, a diffuse growth pattern, microvascular proliferation and/or necrosis [1]. Thus, these tumors have similar histological findings which may cause clinical confusion [4, 5]. BRAFV600 E mutations are seen in 7.7 9.1\% of GBM, $63 \sim 75 \%$ of PXA and $47.4 \sim 57 \%$ of APXA cases $[1,3,6,7]$.

(c) The Author(s). 2020 Open Access This article is distributed under the terms of the Creative Commons Attribution 4.0 International License (http://creativecommons.org/licenses/by/4.0/), which permits unrestricted use, distribution, and 
Although the WHO 2016 classification incorporated molecular testing, diagnoses of anaplastic PXA and GBM are still based on histopathological findings. Here, we present a case of such a complex differential diagnosis of GBM, solely by histology, after taking the clinical course and molecular findings into account.

\section{Case presentation}

A 19-year-old Japanese woman presented with a left temporal headache. Magnetic resonance imaging (MRI) of the brain revealed a well-defined, heterogeneously enhanced tumor in the left temporal lobe, approximately $10 \mathrm{~mm}$ in size, showing high-intensity on T2-weighted images (Fig. 1a, b, c). The patient underwent a left frontal-temporal craniotomy and total resection. These tumor cells had less than 1 mitosis per 10 high-power fields. The primary histopathological diagnosis at that time was low-grade glioma. The patient was discharged after treatment and followed up without any additional treatment. No clear local recurrence was detected during 3 years of follow-up and the patient subsequently elected to stop further medical examinations.

However, 12 years after the initial treatment, the patient returned with a left temporal headache, diplopia and tinnitus. MRI showed a local recurrence of the tumor around the surgical cavity with prominent perifocal edema (Fig. 1d, e, f) and a second resection was performed. Intraoperatively, the tumor was prominently hypervascular, with many feeding arteries from the dura, and a part of the tumor was weakly positive for 5-aminolevuinic acid-based photodynamic diagnosis (PDD). Hematoxylin and eosin (H\&E) staining showed an astrocytic tumor lesion with necrosis, microvascular proliferation, invasion and multiple mitoses ( 5 mitotic counts per 10 highpower fields). No EGBs were observed. (Fig. 2d-g). Immunohistochemical (IHC) stains were positive for oligo2, and tumor cells were retained for INI1 and ATRX staining. Approximately $21 \%$ of these cells were positive for Ki-67 but negative for IDH1-R132H. Furthermore, the specimen from the initial surgery was revisited and the diagnosis was changed to PXA of WHO grade II based on histology showing spindle shaped cells, pleomorphic nucleated cells and EGBs (Fig. 2a, b, c). Reticulin fibers were not stained and CD34 staining was negative in both tumor resections (Fig. 2h-k). As recurrence happened after the PXA resection, APXA was expected due to poorer histological findings; however, the tumor had no characteristic feature of PXA such as EGB, leading to a diagnosis as GBM by histological classification because of nuclear atypia, mitotic activity, a diffuse growth pattern, microvascular proliferation and necrosis (Table 1). The patient received chemotherapy (temozolomide) and conformal radiation therapy accordingly and outpatient follow-ups have detected no obvious symptoms for over 1 year after surgery.
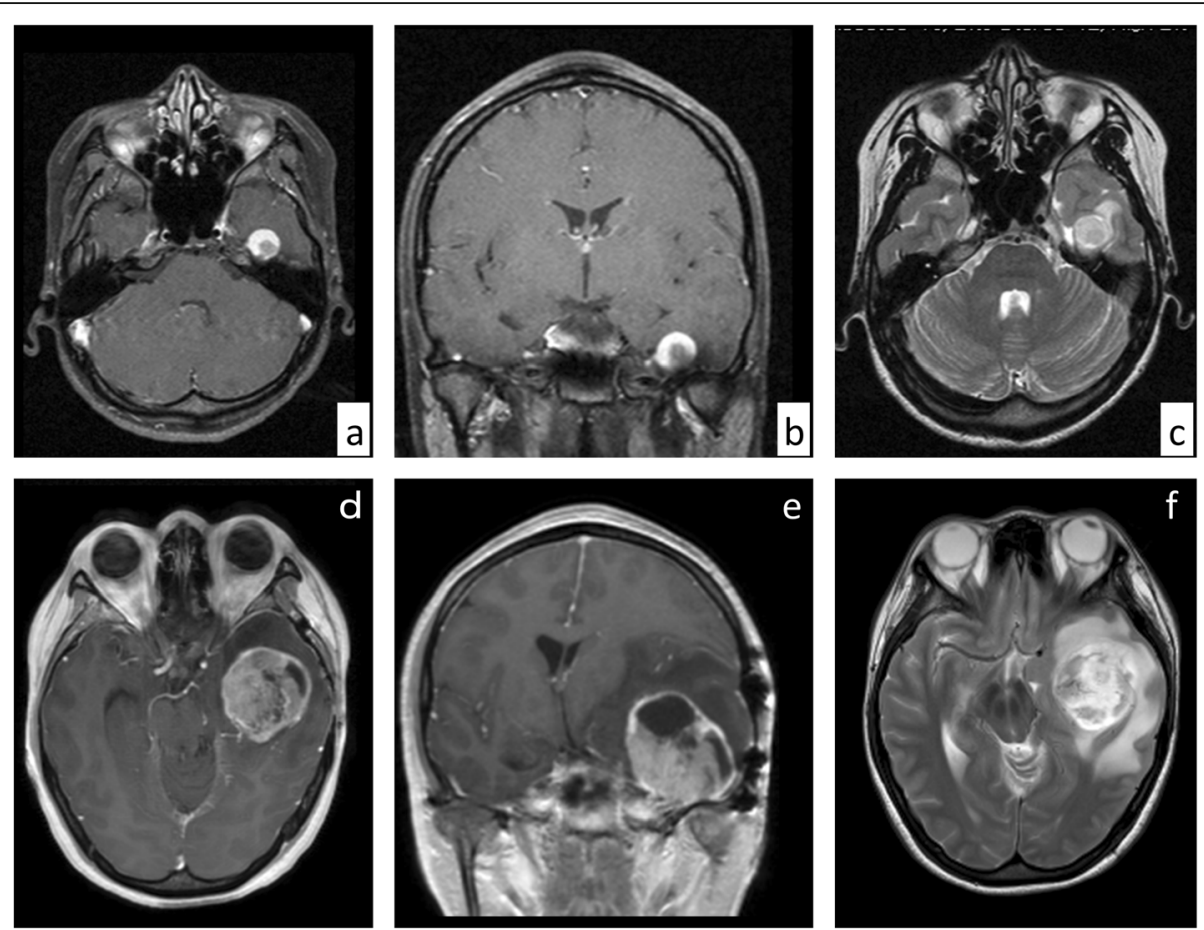

Fig. 1 Neuroimaging along the course of the disease. T1-weighted MR images with gadolinium contrast $(\mathbf{a}, \mathbf{b})$ revealing a well-defined, heterogeneously enhanced tumor in the left temporal lobe, approximately $10 \mathrm{~mm}$ in size, with high intensity on T2-weighted imaging (c). Axial(d) and coronal(e) MR images with gadolinium contrast and axial T2-weighted images (f) showing local recurrence in the surgical cavity 12 years after the initial treatment with a size of approximately $42 \times 45 \times 47 \mathrm{~mm}$ 

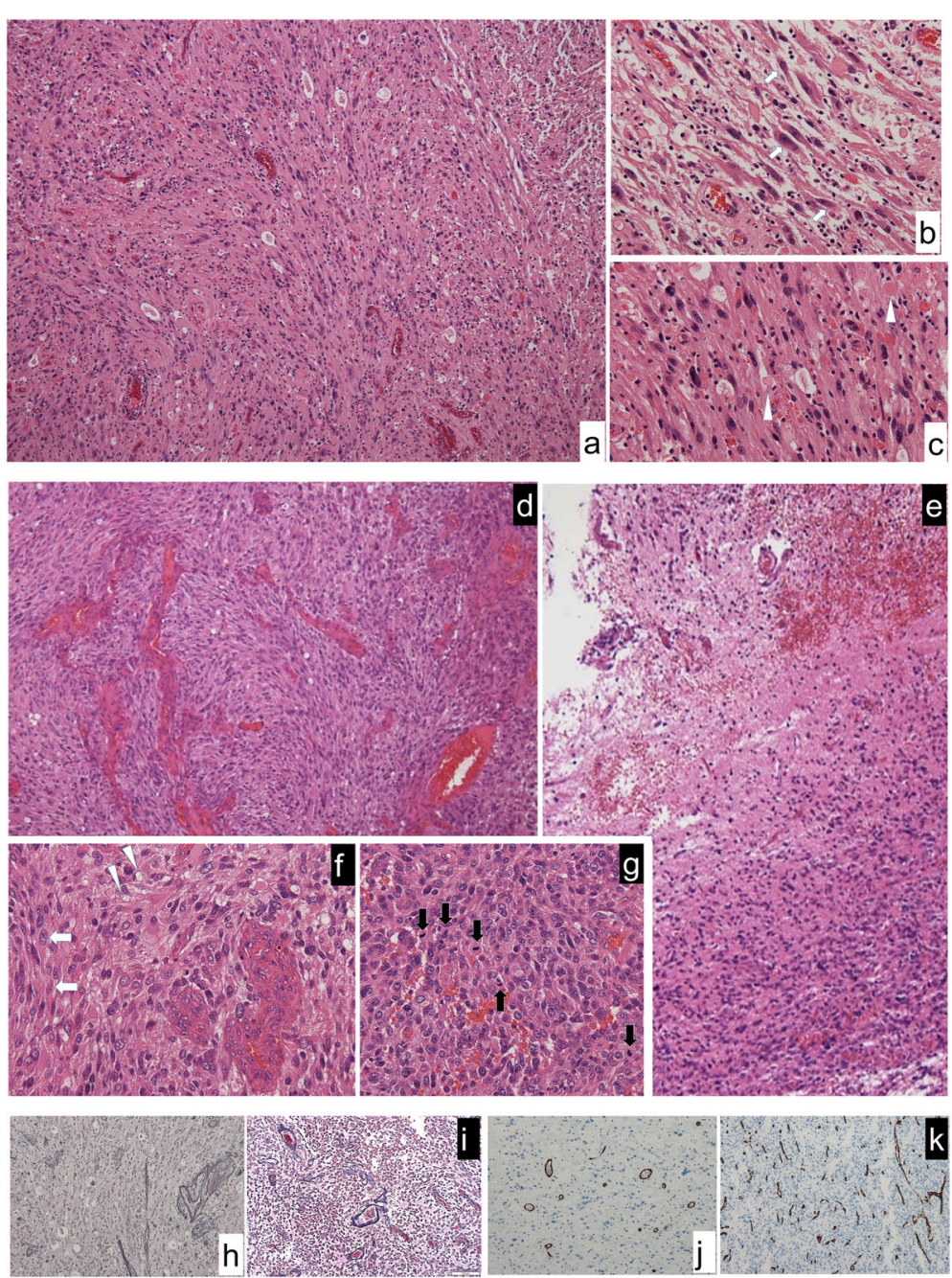

Fig. 2 Microscopic findings of tumors in the initial and recurrent specimens. Spindle cells with nuclear atypia (arrows) with diffusely infiltrating lymphocytes, and eosinophilic granular bodies (arrow heads) as seen in the first resection. (hematoxylin and eosin (H\&E) stain, a $\times 200, \mathbf{b}$ and $\mathbf{c} \times$ 400). In the recurrent specimen, broad necrosis and infiltrating tumor cells can be observed, and massively increased cellularity and prominent atypia are present along the relatively well-defined border line ( $\mathbf{d}$ and $\mathbf{e}$, H\&E stain, $\times 200$ ). With microvascular proliferation, only a small proportion of spindle shape cells (arrows) and xantic cells (arrow heads) can be observed, implying similarity to the previous resection (f stain, $\times 400)$. Multiple mitoses are visible (black arrows), indicating malignancy $(\mathbf{g}$, H\&E stain, $\times 400)$. No reticulin fibers were observed in the first (h) and second (i) specimens (reticulin stain, $\times 200$ ). On CD34 staining, tumor cells were not stained in the first (j) and second (k) specimens (CD34 stain, $\times 200$ )

Additionally, specimens of each resection were sent to the National Cancer Center Research Institute and MLPA (Multiplex Ligation-dependent Probe Amplification) was performed for $1 \mathrm{p}, 19 \mathrm{q}, \mathrm{CDKN} 2 \mathrm{~A}$, IDH1 R132H (c.395G > A), R132C (c.394C > T), IDH2 R172K (c.515G > A), R172M (c.515G > T) and Pyrosequence for IHD1 R132, IDH2 R172, BRAF V600E, H3F3A K27, H3F3A G34, HIST1H3B, TERT C228T, FGFR1 N546, and FGFR1 K656. The analysis detected a BRAF V600E mutation in both the initial and recurrent tumors, with mutant allele findings at 16 and 49\%, respectively. Both $1 p / 19 q$ and CDKN2A were intact in the initial specimen, but a 19q deletion and CDKN2A homozygous deletion were detected in the recurrent specimen. IDH1/IDH2, H3F3A, HIST1H3B, TERT, and FGFR1 were intact in both specimens. From this analysis the tumor was assumed to have been derived from pleomorphic xanthoastrocytoma.

\section{Discussion and conclusions}

In our case, PXA shifted to a more aggressive phenotype with GBM-like features 12 years after the initial surgery. Despite several reports on malignant transformation from PXA to GBM $[2,8,9]$, genetic analyses are absent from most of these cases. 
Table 1 Histological and immunohistological comparison among disease entities (based on Giannini et al. 2016 [1])

\begin{tabular}{|c|c|c|c|c|c|}
\hline & GBM (wild type) & PXA & APXA & $\begin{array}{l}\text { Present case } \\
\text { (1st resection) }\end{array}$ & $\begin{array}{l}\text { Present case } \\
\text { (2nd resection) }\end{array}$ \\
\hline Mitosis (per $10 \mathrm{HPF}$ ) & + & $<5$ & $\geqq 5$ & $<1$ & 5 \\
\hline Necrosis & $-\sim+$ & Rare & $-\sim+$ & - & + \\
\hline MVP & $-\sim+$ & NA & uncommon & - & + \\
\hline Pleomorphism & $-\sim+$ & + & + & + & + \\
\hline IDH mutation & - & - & - & - & - \\
\hline ATRX mutation & - & NA & NA & - & - \\
\hline GFAP & + & + & + & + & + \\
\hline p53 & $-\sim+$ & Variable & NA & $-(<10 \%)$ & $+(15 \%)$ \\
\hline MIB1 & $15-20 \%$ or more & $<2 \sim 5 \%$ & NA & $1 \%$ & $21 \%$ \\
\hline
\end{tabular}

GBM glioblastoma, PXA pleomorphic xanthoastrocytoma, APXA anaplastic PXA, HPF high-power field, NA not available, MVP microvascular proliferation

In the current case, the molecular analysis detected BRAF V600E mutations in both the initial and recurrent tumors but the $19 \mathrm{q}$ and CDKN2A homozygous deletions observed only in the recurrent specimen served as evidence of malignant transformation. The fact that the tumor origin and BRAFV600E mutations were shared in both resections suggest a PXA-derived pathology but PXAlike histology was absent. Histologically, PXA demonstrates spindle shaped cells, pleomorphic nucleated cells, and EGBs while anaplastic APXA is additionally defined when mitoses number 5 or more per high-power field [1]. These findings were absent in the second specimen and, instead, nuclear atypia, mitotic activity, a diffuse growth pattern, microvascular proliferation and necrosis were observed that led to a histological diagnosis of GBM. In GBM, BRAF V600 E mutations are found in $7.7 \sim 9.1 \%$ of cases $[6,10]$ and TERT promoter mutations in $54 \%$ of cases [11] while in APXA, $46.2 \sim 57 \%$ [1, 3], and 23\% [11] of cases, respectively (Table 2). From the point of molecular findings, as BRAF V600E mutation was found in $49 \%$ and TERT promotor mutations $0 \%$ of cells in the second specimen, it was consistent with APXA rather than classic GBM.

Epithelioid GBM (E-GBM), on the other hand, has frequent BRAF V600 E mutations in 16.6 93\% of cases [1214] and E-GBM could arise from PXA, since the BRAF
V600 E mutation is shared among these entities [15]. A recent study showed that concurrent BRAF V600E, TERT promoter mutations and CDKN2A/B homozygous deletions were observed in 50\% of E-GBM cases [13]. Genetic patterning in our case, including a BRAF V600 E mutation with lack of TERT mutation, was comparable to E-GBM (Table 2); however, no histopathological findings consistent with EGBM were observed. Although molecular findings showed overlap with E-GBM, neither resection had a histopathology definitive for diagnosis.

There have been several reports of BRAFV600E-inhibitors as a potential treatment option for gliomas [16-19]. Burger et al. described three patients with recurrent malignant gliomas harboring BRAF V600E mutations in which a complete or nearly complete response to dabrafenib was observed after refractoriness to radiotherapy and alkylating chemotherapy [16]. In cases of acquired resistance to BRAF inhibitors, Nicholas et al. describe two cases of successful treatment with dabrafenib and a MEK inhibitor (trametinib) in relapsed APXA after single agent dabrafenib [17]. Therefore, molecular findings are an important consideration in treatment planning within the context of malignant transformation from PXA even if the histological diagnosis is GBM.

In conclusions, we present a case of malignant transformation of PXA into GBM. Although diagnosed as GBM by histology, the molecular findings implied a PXA origin. In order to properly categorize these disease entities, more integrated criteria, including molecular information, may be needed.

Table 2 Genetic comparison among the different disease entities

\begin{tabular}{lllllll}
\hline & GBM (wild type) & E-GBM & PXA & APXA & $\begin{array}{l}\text { Present case } \\
\text { (1st resection) }\end{array}$ & $\begin{array}{l}\text { Present case } \\
\text { (2nd resection) }\end{array}$ \\
\hline 1p/19q & NA & NA & NA & NA & Intact & $\begin{array}{l}1 p ; \text { intact, } \\
19 q ; \text { deletion }\end{array}$ \\
BRAF V600E & $7.7 \sim 9.1 \%[6,10]$ & $16.6 \sim 93 \%[11,13,14]$ & $63 \sim 75 \%[1,7]$ & $46.2 \sim 57 \%[1,3]$ & Positive (MAF, 16\%) & Positive (MAF, 49\%) \\
CDKN2A/B homozygous deletion & NA & $79 \%[11]$ & $60 \sim 83 \%[2,8]$ & $93 \%[7]$ & Intact & Positive \\
TERT promoter mutation & $54 \%[11]$ & $71 \%[11]$ & $4 \%[11]$ & $23 \%[11]$ & Negative & Negative \\
\hline
\end{tabular}




\section{Abbreviations}

APXA: Anaplastic pleomorphic xanthoastrocytoma; EGB: Eosinophilic granular body; E-GBM: Epithelioid glioblastoma; GBM: Glioblastoma; H\&E: Hematoxylin and eosin (staining); HPF: High-power field; IHC: Immunohistochemical (staining); MAF: Mutant allele frequency; MVP: Microvascular proliferation; NA: Not available; PDD: Photodynamic diagnosis; PXA: Pleomorphic xanthoastrocytoma; WHO: World Health Organization

\section{Acknowledgements}

The authors are grateful to Dr. Ichimura of Division of Brain Tumor Translational Research, National Cancer Center Research Institute for the helpful molecular analyses. We also thank Dr. Bryan J. Mathis of the Medical English Communications Center of the Faculty of Medicine, University of Tsukuba, for native English revision.

\section{Authors' contributions}

Design of the work - NW, El, MS, AM; acquisition and analysis - NW, El, HK, NS, $\mathrm{MM}$; interpretation of data - NW, El, NS, MS, drafting and revision of the manuscript - NW, El, AZ. All authors agreed with their authorship and approved the submitted version of the manuscript.

\section{Funding}

No funding was received for this research.

\section{Availability of data and materials}

The datasets used and/or analyzed during the current study are available from the corresponding authors on reasonable request.

\section{Ethics approval and consent to participate}

The current study has been performed in accordance with the Declaration of Helsinki. For case reports, ethics committee approval is not necessary at our organization. Informed consent was obtained from the patient included in the study.

\section{Consent for publication}

Written informed consent was obtained from the patient included in the study.

\section{Competing interests}

The authors declare that they have no competing interests.

\section{Author details}

'Departments of Neurosurgery, Faculty of Medicine, University of Tsukuba, 1-1-1 Tennodai, Tsukuba, Ibaraki, Japan. ${ }^{2}$ Departments of Diagnostic Pathology, Faculty of Medicine, University of Tsukuba, Tsukuba, Ibaraki, Japan. ${ }^{3}$ Central Laboratory, Hachioji Medical Center, Tokyo Medical University, Tokyo, Japan.

Received: 9 July 2019 Accepted: 6 January 2020

\section{Published online: 15 January 2020}

\section{References}

1. Giannini C, Paulus W, Louis DN, Liberski PP, Figarella-Branger D, Capper D. Pleomorphic xanthoastrocytoma. In: Louis DN, Ohgaki H, Wiestler OD, Cavenee WK, editors. WHO classification of Tumours of the central nervous system, vol. 28-45. Lyon: International Agency for Research on Cancer (IARC); 2016. p. 94-9.

2. Oh T, Kaur G, Madden M, Bloch O, Parsa AT. Pleomorphic xanthoastrocytomas: institutional experience of 18 patients. J Clin Neurosci. 2014;21:1767-72.

3. Ida CM, Rodriguez FJ, Burger PC, Caron AA, Jenkins SM, Spears GM, et al. Pleomorphic Xanthoastrocytoma: natural history and long-term follow-up. Brain Pathol. 2015;25:575-86.

4. Yamada SM, Murakami H, Tomita Y, Nakane M, Shibui S, Takahashi M, et al. Glioblastoma multiforme versus pleomorphic xanthoastrocytoma with anaplastic features in the pathological diagnosis: a case report. Diagn Pathol. 2016:11:65.

5. Binesh F, Akhavan A, Navabii H. Pleomorphic xanthoastrocytoma with malignant transformation and multiple recurrences in an Iranian girl. BMJ Case Rep. 2012;2012:bcr1220115372.
6. Takahashi Y, Akahane T, Sawada T, Ikeda H, Tempaku A, Yamauchi S, et al. Adult classical glioblastoma with a BRAF V600E mutation. World I Surg Oncol. 2015;13:100.

7. Vaubel RA, Caron AA, Yamada S, Decker PA, Eckel Passow JE, Rodriguez FJ, et al. Recurrent copy number alterations in low-grade and anaplastic pleomorphic xanthoastrocytoma with and without BRAF V600E mutation. Brain Pathol. 2018;28:172-82.

8. Vu TM, Liubinas SV, Gonzales M, Drummond KJ. Malignant potential of pleomorphic xanthoastrocytoma. J Clin Neurosci. 2012;19:12-20.

9. Singla N, Kapoor A, Radotra BD, Chatterjee D. Malignant conversion to Glioblastoma in Neurofibromatosis type l-associated pleomorphic Xanthoastrocytoma: unknown predictors of favorable outcome. Asian J Neurosurg. 2018;13:826-9.

10. Dahiya S, Emnett RJ, Haydon DH, Leonard JR, Phillips JJ, Perry A, et al. BRAF-V600E mutation in pediatric and adult glioblastoma. Neuro-Oncology. 2014:16:318-9.

11. Koelsche C, Sahm F, Capper D, Reuss D, Sturm D, Jones DT, et al. Distribution of TERT promoter mutations in pediatric and adult tumors of the nervous system. Acta Neuropathol. 2013;126:907-15.

12. Kleinschmidt-DeMasters BK, Aisner DL, Birks DK, Foreman NK. Epithelioid GBMs show a high percentage of BRAF V600E mutation. Am J Surg Pathol. 2013;37:685-98.

13. Nakajima N, Nobusawa S, Nakata S, Nakada M, Yamazaki T, Matsumura N, et al. BRAF V600E, TERT promoter mutations and CDKN2A/B homozygous deletions are frequent in epithelioid glioblastomas: a histological and molecular analysis focusing on intratumoral heterogeneity. Brain Pathol. 2018:28:663-73.

14. Tosuner Z, Geçer MÖ, Hatiboğlu MA, Abdallah A, Turna S. BRAF V600E mutation and BRAF VE1 immunoexpression profiles in different types of glioblastoma. Oncol Lett. 2018;16:2402-8.

15. Tanaka S, Nakada M, Nobusawa S, Suzuki SO, Sabit H, Miyashita K, et al. Epithelioid glioblastoma arising from pleomorphic xanthoastrocytoma with the BRAF V600E mutation. Brain Tumor Pathol. 2014;31:172-6.

16. Burger MC, Ronellenfitsch MW, Lorenz NI, Wagner M, Voss M, Capper D, et al. Dabrafenib in patients with recurrent, BRAF V600E mutated malignant glioma and leptomeningeal disease. Oncol Rep. 2017;38:3291-6.

17. Nicholas FB, Thomas C, Nell K, Paul M. Dabrafenib and trametinib in BRAFV600E mutated glioma. CNS Oncol. 2017;6:291-6.

18. Del Bufalo F, Ceglie G, Cacchione A, Alessi I, Colafati GS, Carai A, et al. BRAF V600E inhibitor (Vemurafenib) for BRAF V600E mutated low grade Gliomas. Front Oncol. 2018;8:526.

19. Brown NF, Carter T, Mulholland P. Dabrafenib in BRAFV600mutated anaplastic pleomorphic xanthoastrocytoma. CNS Oncol. 2017;6:5-9.

\section{Publisher's Note}

Springer Nature remains neutral with regard to jurisdictional claims in published maps and institutional affiliations.

\section{Ready to submit your research? Choose BMC and benefit from:}

- fast, convenient online submission

- thorough peer review by experienced researchers in your field

- rapid publication on acceptance

- support for research data, including large and complex data types

- gold Open Access which fosters wider collaboration and increased citations

- maximum visibility for your research: over $100 \mathrm{M}$ website views per year

At BMC, research is always in progress.

Learn more biomedcentral.com/submissions 